\title{
Charge-sign dependence in the solar modulation during the solar cycle 23
}

\author{
Shoko Miyake*1 $\dagger$ \\ ${ }^{1}$ National Institute of Technology, Ibaraki College, Ibaraki, Japan
}

\begin{abstract}
Charge-sign dependence of the solar modulation of galactic cosmic ray (GCR) protons and antiprotons is numerically investigated. The calculations are performed by considering the gradientcurvature drift motion and fully anisotropic diffusion. We also assume the variations of the solar wind speed, the strength of the heliospheric magnetic field, and the tilt angle of the heliospheric current sheet. We calculate the energy spectra of the GCR protons and antiprotons during the solar cycle 23, and quantitatively investigate the charge-sign dependence of the solar modulation by comparing our results with the observations by BESS (Balloon-borne Experiment with a Superconducting Spectrometer) and PAMELA (Payload for Antimatter Matter Exploration and Light-nuclei Astrophysics).
\end{abstract}

35th International Cosmic Ray Conference - ICRC2017

10-20 July, 2017

Bexco, Busan, Korea

\footnotetext{
* Speaker.

${ }^{\dagger}$ E-mail: miyakesk@ee.ibaraki-ct.ac.jp
} 


\section{Introduction}

Gradient-curvature drift motion of the GCRs in the heliosphere causes a charge-sign dependence of the solar modulation, such as the 22-years variations of the flux of GCRs that has a flat-top and sharp-top time profile. In this study we develope the numerical model for the solar modulation on the basis of the drift model to study quantitatively the charge-sign dependence. In the solar cycle 23, the long term observations of multiple GCRs, such as protons, heliums, deuterons, and those antimatters, have been performed by BESS (e.g. Sakai et al. [四) and PAMELA (e.g. Adriani et al. [వ] ). Because these observations entirely cover the solar minimum, the solar maximum, and the solar magnetic polarity reversal, the fluxes of GCRs measured by these observations provide a crucial test of a charge-sign dependence of the solar modulation. We thus calculate the energy spectra of GCR protons and antiprotons during the solar cycle 23 and compare our results with that measured by these observations. The details of our modulation model and the heliospheric model are described in section 2 and 3, respectively. In section 4, we present our results of the energy spectra of GCR protons and the antiproton/proton ratio during the solar cycle 23.

\section{Modulation Model}

We numerically investigate the propagation of GCR protons and antiprotons in the heliosphere. Our calculation is based on the equivalence of a coupled set of SDEs and the Parker's convection-

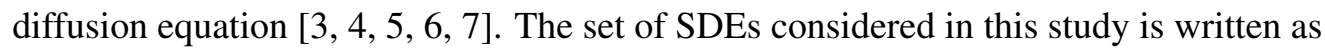

$$
\begin{aligned}
& d \mathbf{r}=\left(\nabla \cdot \kappa+\mathbf{V}_{\mathrm{sw}}+\mathbf{V}_{\mathrm{drift}}\right) d t+\sum_{s} \sigma_{s} d W_{s}(t), \\
& d p=-\frac{1}{3} p\left(\nabla \cdot \mathbf{V}_{\mathrm{sw}}\right) d t
\end{aligned}
$$

where $\mathbf{r}$ and $p$ indicate the position and momentum of pseudo-particle respectively, $t$ is the time, $\kappa$ is the spatial diffusion coefficient tensor, $\mathbf{V}_{\mathrm{sw}}$ is the velocity of the solar wind, $\mathbf{V}_{\text {drift }}$ is the velocity of the gradient-curvature drift motion, $\sum_{s} \sigma_{s}^{\mu} \sigma_{s}^{v}=2 \kappa^{\mu v}$, and $d W_{s}$ is the Wiener process given by the Gaussian distribution. Here we calculate the drift motion along the heliospheric current sheet (HCS) by using an approximate function proposed by Burger and Potgieter [ [ $]$ ]. We consider the fully anisotropic diffusion distinguishing three diffusion axes in the magnetic field coordinate system, as follows:

$$
\begin{aligned}
& \kappa_{\|}=\left(\kappa_{\|}\right)_{0} \beta\left(\frac{p}{1 \mathrm{GeV} / \mathrm{C}}\right)^{\delta}\left(\frac{B_{\mathrm{e}}}{B}\right)^{\eta_{\|}}, \\
& \kappa_{\perp_{1}}=\left(\kappa_{\perp_{1}}\right)_{0} \beta\left(\frac{p}{1 \mathrm{GeV} / \mathrm{C}}\right)^{\delta}\left(\frac{B_{\mathrm{e}}}{B}\right)^{\eta_{\perp_{1}}}, \\
& \kappa_{\perp_{2}}=\left(\kappa_{\perp_{2}}\right)_{0} \beta\left(\frac{p}{1 \mathrm{GeV} / \mathrm{C}}\right)^{\delta}\left(\frac{B_{\mathrm{e}}}{B}\right)^{\eta_{\perp_{2}}},
\end{aligned}
$$

where $\beta$ is the velocity of particle relative to the velocity of light and $B_{\mathrm{e}}$ is the strength of the heliospheric magnetic field (HMF) near the Earth. We define $\kappa_{\perp_{1}}$ and $\kappa_{\perp_{2}}$ as the diffusion coefficient of the heliospheric polar angle direction and that of the other direction perpendicular to the HMF, respectively. $\left(\kappa_{\|}\right)_{0},\left(\kappa_{\perp_{1}}\right)_{0},\left(\kappa_{\perp_{2}}\right)_{0}, \delta, \eta_{\|}, \eta_{\perp_{1}}$, and $\eta_{\perp_{2}}$ are regarded as free parameters in our model. 


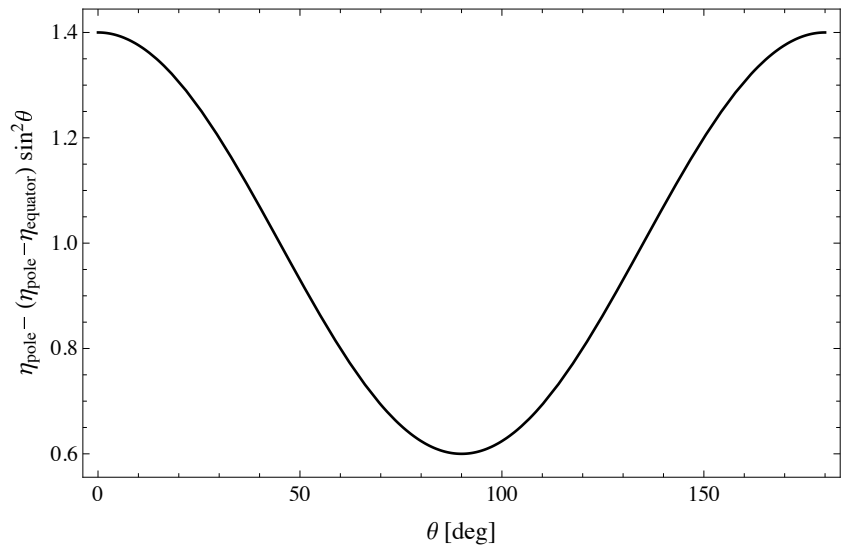

Figure 1: Modeled profile of the power-law index of the magnetic strength dependence for the diffusion coefficient of the polar angle direction.

We found following values that can largely reproduce the energy spectra of protons and antiprotons by iterative searching: $\left(\kappa_{\|}\right)_{0}=1 \times 10^{22} \mathrm{~cm}^{2} / \mathrm{s} ;\left(\kappa_{\perp_{1}}\right)_{0}=1 \times 10^{20} \mathrm{~cm}^{2} / \mathrm{s} ;\left(\kappa_{\perp_{2}}\right)_{0}=2 \times 10^{20} \mathrm{~cm}^{2} / \mathrm{s}$; $\delta=1.0 ; \eta_{\|}=1.0 ; \eta_{\perp_{1}}=\eta_{\mathrm{p}}-\left(\eta_{\mathrm{p}}-\eta_{\mathrm{e}}\right) \sin ^{2} \theta=1.4-(1.4-0.6) \sin ^{2} \theta ;$ and $\eta_{\perp_{2}}=1.0 . \eta_{\mathrm{p}}$ and $\eta_{\mathrm{e}}$ are the power-law indexes for the magnetic dependences at the pole and the equator in the heliosphere, respectively. By describing $\eta_{\perp_{1}}$ as a function of $\theta$ as shown in Figure $\mathbb{W}$, we take into account the latitudinal dependence of the diffusion coefficient in the polar angle direction.

The distribution function at the Earth $f_{\mathrm{e}}\left(p_{0}\right)$ is described as a convolution of the distribution function at the heliospheric boundary $f_{\text {lism }}(p)$ with the normalized transition probability $F\left(p_{0}, r_{0} \mid p, r\right)$ obtained by our calculation, namely

$$
f_{\mathrm{e}}\left(p_{0}\right)=\int f_{\text {lism }}(p) F\left(p_{0}, r_{0} \mid p, r_{\text {out }}\right) d p .
$$

We adopt the following local interstellar spectrum (LIS) of GCR protons and antiprotons at the heliospheric boundary,

$$
\begin{aligned}
& J_{p}\left(E_{\mathrm{k}}\right)=16.0\left(1+\frac{4.2}{E_{\mathrm{k}}^{1.22}}+\frac{1.3}{E_{\mathrm{k}}^{2.8}}+\frac{0.0087}{E_{\mathrm{k}}^{4.32}}\right)^{-1} E_{\mathrm{k}}^{-2.73}, \\
& J_{\bar{p}}\left(E_{\mathrm{k}}\right)=3.0 \exp \left[-\exp \left\{1.59-1.65 \log E_{\mathrm{k}}-0.83\left(\log E_{\mathrm{k}}\right)^{2}-0.21\left(\log E_{\mathrm{k}}\right)^{3}\right\}\right] E_{\mathrm{k}}^{-2.73},
\end{aligned}
$$

where $E_{\mathrm{k}}$ is the kinetic energy of GCR at the heliospheric boundary. The LIS of GCR protons $J_{p}$ has a similar energy dependence with a LIS that is in agreement with the flux of GCR protons measured by Voyager 1 outside of the heliosphere [Q, 四], though we modified it so that the flux of the high energy protons consists with the data measured by BESS-TeV [ए]]. The LIS of GCR antiprotons $J_{\bar{p}}$ that has the same power-law index at high energy region is assumed [ए]].

\section{Heliospheric Model}

In this study, we consider the variations of $V_{\mathrm{sw}}, B_{\mathrm{e}}$, and the tilt angle of the $\operatorname{HCS} \alpha . V_{\mathrm{sw}}$ is assumed to be a constant within the heliospheric boundary. We adopt the Parker-Spiral HMF whose 

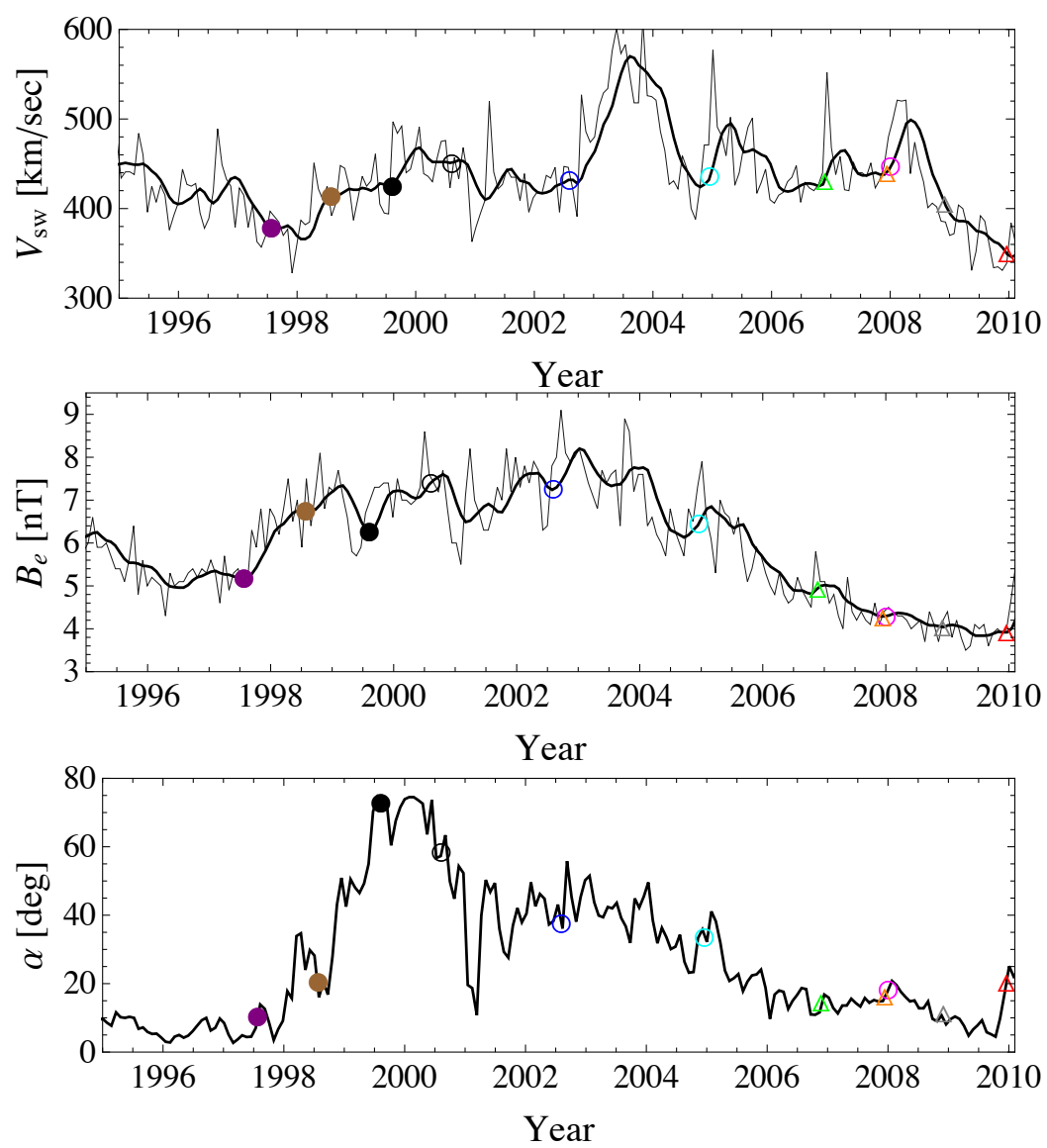

Figure 2: Modeled and observed profiles of the solar wind velocity (top panel), the strength of the HMF close to the Earth (middle panel), and the tilt angle of the HCS (bottom panel). Gray lines and Black lines show the observed values and the values used in our calculation, respectively. The markers indicate the values adopted to make a comparison of our calculation with the observations by BESS [ए3], ए4]] and PAMELA []]: purple filled circle, for BESS (1997); brown filled circle, for BESS (1998); black filled circle, for BESS (1999); black open circle, for BESS (2000); blue open circle, for BESS (2002); cyan open circle, for BESS-Polar I (2004); magenta open circle, for BESS-Polar II (2007); green open triangle, for PAMELA (2006); orange open triangle, for PAMELA (2007); gray open triangle, for PAMELA (2008); and red open triangle, for PAMELA (2009).

strength is determined by $B_{\mathrm{e}}$ and the wavy HCS whose structure is determined by $\alpha$ and the rotation of the Sun. We took 150-day averaged values of $V_{\text {sw }}$ and $B_{\mathrm{e}}$ obtained from the GSFC/SPDF OMNIWeb interface [ㄷ] shown in Figure $\square$, considering the constant time lag between the observations close to the Sun and near the heliospheric boundary. We also assume $\alpha$ changing with both time and heliospheric radius, which is obtained from the Wilcox Solar Observatory [ए]]. We postulate that the HMF polarity changes from positive to negative in 2000.2 , in which the maximum value of the HCS tilt angle is observed.

\section{Result and Discussion}

If the product of the charge of particles, $q$, by the polarity of the $\mathrm{HMF}, A$, is negative, $q A<0$, 


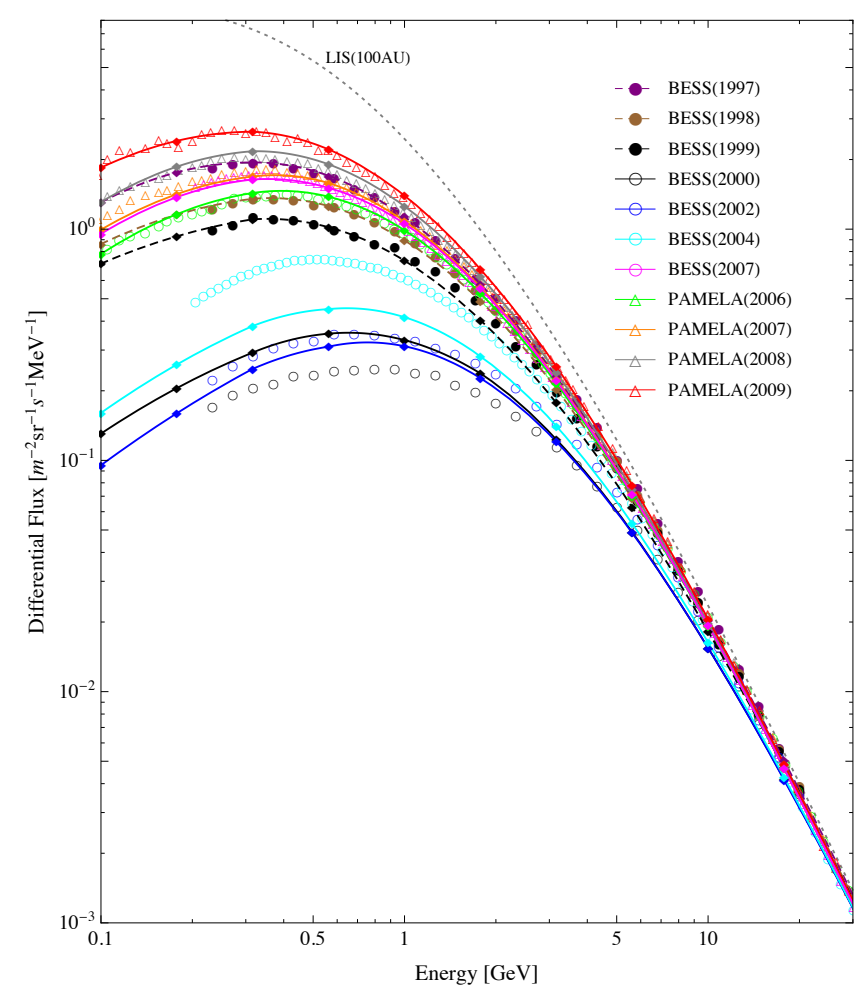

Figure 3: Calculated and observed energy spectra of GCR protons. Solid or dashed lines indicate the energy spectra of GCR protons obtained by our calculation. Gray dotted line shows the LIS considered in our

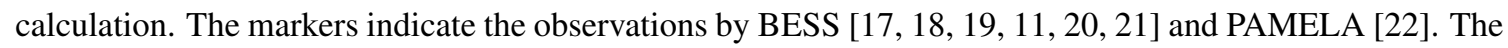
caption of these markers is the same as Figure $\square$.

the particles propagate along the HCS from the heliospheric boundary toward the Earth, whereas in $q A>0$ the particles propagate from the polar region of the heliosphere. This is because of the gradient-curvature drift motion in the HMF and it leads to the charge-sign dependence of the solar modulation. Our results of the energy spectra of the GCR protons and antiprotons show such a charge-sign dependence as shown in Figure $B$ and Figure 4 . Figure 3 shows the energy spectra of the GCR protons during 1997-2009. We can find that the energy spectra in $A<0$ (solid lines) strongly change than that in $A>0$ (dashed lines). This is because the effect of the HCS tilt angle, namely the structure of the HCS, on the GCR flux in $q A<0$ is larger than that in $q A>0$ (e.g. Miyake et al. [ [D]). The flux at the solar minimum in $q A<0$ larger than that in $q A>0$ is caused by the drift velocity along the HCS larger than the convection velocity and the typical diffusion velocity of the charged particle in the heliosphere [[23, [8]. Rapid change of the antiproton/proton ratio observed by BESS during 1999-2000 could be explained by the charge-sign dependence of the solar modulation and the polarity reversal of the HMF happened in these period. Our calculation succeeded to reproduce this rapid change of the antiproton/proton ratio, as shown in Figure $⿴$.

In this study, we consider that the diffusion coefficient of the heliospheric polar angle direction at the polar region is larger than that at the equatorial region. This feature of the diffusion coefficient of the polar angle direction is consistent with the findings by the studies of the latitudinal gradients of the GCR protons measured by the Ulysses spacecraft [24], though the details of the diffusion 


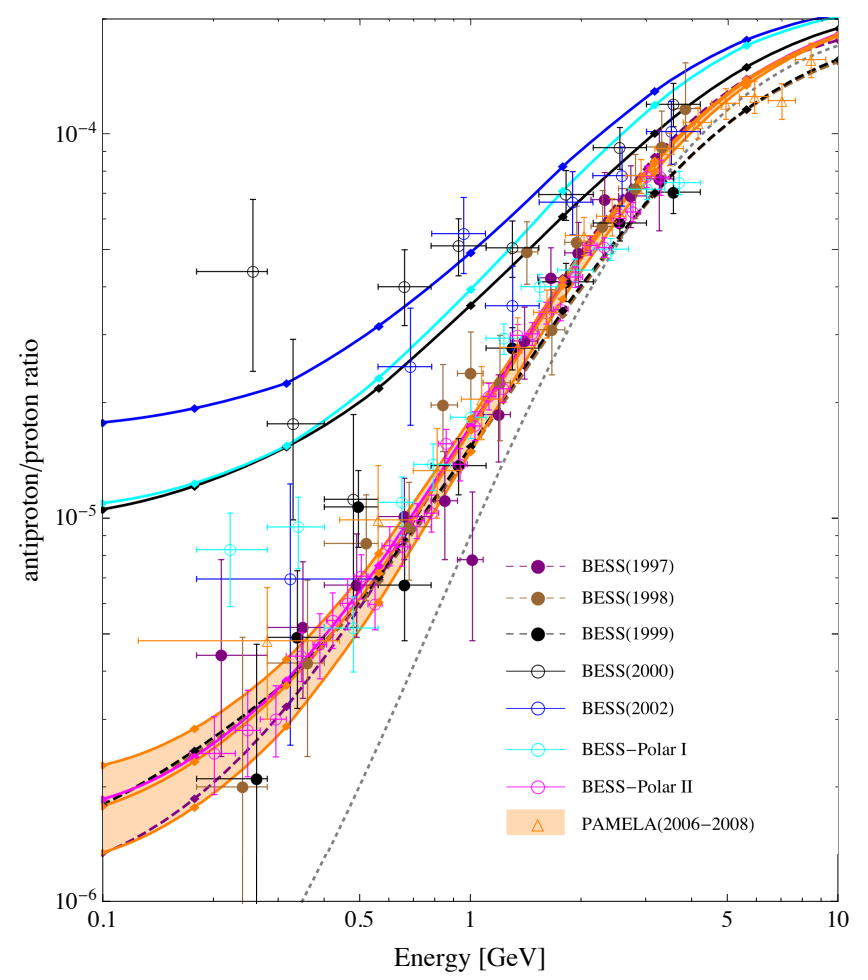

Figure 4: Calculated and observed GCR $\bar{p} / p$ ratios. The caption of lines and markers is the same as Figure [3.

coefficient assumed by Burger and Potgieter [24] is different with that considered in our model. The large diffusion coefficient of the polar angle direction near the polar region indirectly reduces the drift effects in $q A>0$. This may suggest the Fisk-type HMF caused by the differential rotation of the Sun $[25,[26]$, in which the structure of the magnetic field is different with that of the ParkerSpiral HMF at the polar region. As other possible reason of the large diffusion coefficient of the polar angle direction, one could expect the magnetic field near the pole that is dominated by the randomly-oriented transverse magnetic fields with magnitude much larger than that of the ParkerSpiral HMF [27].

Our results of the antiproton/proton ratios show good agreement with the observations by BESS and PAMELA except for a discrepancy in 2004. This discrepancy between our result and the observation in 2004 is because our result of the energy spectrum of GCR protons is lower than the observation, as shown in Figure [3. We also found that there are discrepancies between our results of the energy spectra of GCR antiprotons and the energy spectra observed by BESS in 1998 and 1999, although we do not show the details in this paper. The year 2004 for the GCR protons and the years 1998 and 1999 for the GCR antiprotons correspond to the transition period for $q A<0$. This may imply that there is any physical reason that is not considered in our model for the transition period for $q A<0$. The detailed discussion of the possible reasons for this discrepancy of the flux at the transition period for $q A<0$ will be presented in another paper. 


\section{Acknowledgments}

We express our sincere thanks for the invaluable comments for this work of J. W. Mitchel, K. Sakai, M. Sasaki, J. Krizmanic, A. Moiseev, Y. Akaike, and S. Yanagita. The numerical calculation has been made by using the Advanced Data Analytics Platform (ADAPT) at NASA Center for Climate Simulation. The solar wind velocity and HMF magnitude data were obtained from the GSFC/SPDF OMNIWeb interface at http://omniweb.gsfc.nasa.gov. The data of the tilt angle of the HCS was obtained via the web site http://wso.stanford.edu at 2016:06:02_18:34:11 PDT courtesy of J.T. Hoeksema. We also express our thanks for providing these computer resources or data. S.M.'s work is partially supported by JSPS KAKENHI Grant Number JP26800145.

\section{References}

[1] K. Sakai, K. Abe, H. Fuke, S. Haino, T. Hams, et al., Measurement of the cosmic-ray antiproton spectrum in the range 0.12 to $0.4 \mathrm{GeV}$ with BESS-Polar II, The 35th International Cosmic Ray Conference (Busan, Korea) (2017) CRD086.

[2] O. Adriani, G. C. Barbarino, G. A. Bazilevskaya, R. Bellotti, M. Boezio, et al., Time dependence of the proton flux measured by PAMELA during the 2006 July - 2009 December solar minimum, The Astrophys. J. 765 (2013) 91.

[3] Y. Yamada, S. Yanagita, and T. Yoshida, A stochastic view of the solar modulation phenomena of cosmic rays, Geophys. Res. Lett. 25 (1998), no. 13 2353-2356.

[4] M. Zhang, A Markov stochastic process theory of cosmic-ray modulation, Astrophys. J. 513 (1999) 409-420.

[5] C. Pei, J. W. Bieber, R. A. Burger, and J. Clem, A general time-dependent stochastic method for solving Parker's transport equation in spherical coordinates, J. Geophys. Res. 115 (2010) A12107.

[6] R. D. Strauss, M. S. Potgieter, I. Büsching, and A. Kopp, Modelling heliospheric current sheet drift in stochastic cosmic ray transport models, Astrophys. Space Sci. 339 (2012) 223-236.

[7] S. Miyake, R. Kataoka, and T. Sato, Cosmic ray modulation and radiation dose of aircrews during the solar cycle 24/25, Space Weather 15 (2017) 589-605.

[8] R. A. Burger and M. S. Potgieter, The calculation of neutral sheet drift in two-dimensional cosmic-ray modulation models, The Astrophys. J. 339 (1989) 501-511.

[9] W. R. Webber and P. R. Higbie, Galactic propagation of cosmic ray nuclei in a model with an increasing diffusion coefficient at low rigidities: A comparison of the new interstellar spectra with Voyager data in the outer heliosphere, J. Geophys. Res. 114 (2009) A02103.

[10] W. R. Webber and P. R. Higbie, What Voyager cosmic ray data in the outer heliosphere tells us about ${ }^{10}$ Be production in the Earth's polar atmosphere in the recent past, J. Geophys. Res. 115 (2010) A05102.

[11] S. Haino, T. Sanuki, K. Abe, K. Anraku, Y. Asaoka, et al., Measurements of primary and atmospheric cosmic-ray spectra with the BESS-TeV spectrometer, Phys. Lett. B 594 (2004) 35-46.

[12] M. Aguilar, L. A. Cavasonza, B. Alpat, G. Ambrosi, L. Arruda, et al., Antiproton flux, antiproton-to-proton flux ratio, and properties of elementary particle fluxes in primary cosmic rays measured with the Alpha Magnetic Spectrometer on the international space station, Phys. Rev. Lett. 117 (2016) 091103. 
[13] Y. Shikaze, S. Haino, K. Abe, H. Fuke, T. Hams, et al., Measurements of 0.2 - 20 GeV/n cosmic-ray proton and helium spectra from 1997 through 2002 with the BESS spectrometer, Astropart. Phys. 28 (2007) 154-167.

[14] K. Abe, H. Fuke, S. Haino, T. Hams, M. Hasegawa, et al., Measurements of cosmic-ray proton and helium spectra from the BESS-Polar long-duration balloon flights over antarctica, The Astrophys. J. 822 (2016) 65.

[15] J. H. King and N. E. Papitashvili, Solar wind spatial scales in and comparisons of hourly wind and ACE plasma and magnetic field data, J. Geophys. Res. 110 (2005) A02104.

[16] J. T. Hoeksema, The large-scale structure of the heliospheric current sheet during the Ulysses epoch, Space Sci. Rev. 72 (1995) 137-148.

[17] S. Orito, T. Maeno, H. Matsunaga, K. Abe, K. Anraku, et al., Precision measurement of cosmic-ray antiproton spectrum, Phys. Rev. Lett. 84 (2000) 1078-1081.

[18] T. Maeno, S. Orito, H. Matsunaga, K. Abe, K. Anraku, et al., Successive measurements of cosmic-ray antiproton spectrum in a positive phase of the solar cycle, Astropart. Phys. 16 (2001) 121-128.

[19] Y. Asaoka, Y. Shikaze, K. Abe, K. Anraku, M. Fujikawa, et al., Measurements of cosmic-ray low-energy antiproton and proton spectra in a transient period of solar field reversal, Phys. Rev. Lett. 88 (2002) 051101.

[20] K. Abe, H. Fuke, S. Haino, T. Hams, A. Itazaki, et al., Measurement of the cosmic-ray low-energy antiproton apectrum with the first BESS-Polar antarctic flight, Phys. Lett. B 670 (2008) 103-108.

[21] K. Abe, H. Huke, S. Haino, T. Hams, M. Hasegawa, et al., Measurement of the cosmic-ray antiproton spectrum at solar minimum with a long-duration balloon flight over antarctica, Phys. Rev. Lett. 108 (2012) 051102.

[22] O. Adriani, G. C. Barbarino, G. A. Bazilevskaya, R. Bellotti, M. Boezio, et al., PAMELA results on the cosmic-ray antiproton flux from $60 \mathrm{MeV}$ to $180 \mathrm{GeV}$ in kinetic energy, Phys. Rev. Lett. 105 (2010) 121101.

[23] J. Kóta and J. R. Jokipii, Effects of drift on the transport of cosmic rays. VI. a three-dimensional model including diffusion, The Aptrophys. J. 265 (1983) 573-581.

[24] R. A. Burger and M. S. Potgieter, Rigidity dependence of cosmic ray proton latitudinal gradients measured by the Ulysses spacecraft: Implications for the diffusion tensor, Journal of Geophys. Res. 105 (2000) A12.

[25] L. A. Fisk, Motion of the footpoints of heliospheric magnetic field lines at the sun: Implications for recurrent energetic particle events at high heliographic latitudes, Journal of Geophys. Res. 101 (1996), no. A7 15,547-15,553.

[26] L. A. Fisk, T. H. Zurbuchen, and N. A. Schwadron, On the coronal magnetic field: Consequences of large-scale motions, The Astrophys. J. 521 (1999) 868-877.

[27] J. R. Jokipii and J. Kóta, The polar heliospheric magnetic field, Geophys. Res. Lett. 1 (1989) 1-4. 